\title{
Investigation on the role of atomization air mixing on the spray characteristics of air assisted atomizer
}

\author{
Noritsune Kawaharada*1, Markus Höltermann ${ }^{1}$, Jan Wichmar ${ }^{1}$, Friedrich Dinkelacker ${ }^{1}$ \\ ${ }^{1}$ Institute of Technical Combustion, Leibniz Universität Hannover, Germany \\ *Corresponding author: kawaharada@itv.uni-hannover.de
}

\begin{abstract}
Air assisted atomizers are widely used for various purposes, for example coating processes, medical processes, and sprinkler. However, the spray development processes, especially the breakup phenomena are not fully understood yet. Generally, the main breakup of the air assisted atomizer depends on whether the mixing is internal or external. The border between these processes is not clear. In order to study changes in the spray configuration due to the way of mixing, the spray was investigated for the transition from internal to external mixing. Therefore, an air assisted atomizer which allows adjusting the distance between injector to cover was used. The atomizer consists of two components, which are a liquid injector and an injector cover with orifice. The atomization air flows through the gap between the injector and the cover. The position of cover orifice is able to be traversed from the position fully attached to the injector to several millimetre distances from the injector nozzle. In this study, the water spray characteristics depending on the air and liquid mixture position were investigated experimentally by two optical measurement techniques.

Imaging techniques were used for taking the spray structure as well as liquid core in near nozzle field. The water mass flow, atomization air mass flow, and the cover position were changed and their effects on the spray were investigated. Phase Doppler Anemometer (PDA) was applied for measurement of velocity and size of droplets in water spray which injected into the atmosphere. The measurement positions were set at planes which located on 20 and $40 \mathrm{~mm}$ downstream from the nozzle orifice. For each downstream position, radial profiles of the spray pattern were measured on one air to water flow rate condition. The cover positions were changed and the differences on the droplet velocity and diameter as a function of radial distance on each nozzle cover positions were discussed. Also, high resolution direct imaging technique has been developed and applied for measurement of size of droplets. The results from these measurement techniques were compared and they correspond well at the high air to water flow rate conditions. At the low air to water flow rate condition, the agreement of the results of these measurements became lower. It might be caused by the bouncing unspherical droplets.
\end{abstract}

\section{Keywords}

Air-assisted-atomizer, Laser diagnostics, Direct imaging techniques, Atomization process, Atmospheric-pressure

\section{Introduction}

The twin fluid atomizer can be applied for various purposes for example coating processes, medical processes, and sprinkler. Most of twin-fluid nozzles were categorized to air assisted or air blast. The main difference is velocity of air flow, and the air velocity of air assisted atomizer is higher than that of air blast atomizer. The effect of mass flow rate of air and water on droplet diameter from air blast atomizer was investigated by Nukiyama etal. [1] in 1939. Lefebvre [2] summarized and explained about factors which influenced to mean droplet size. However, the spray development processes, especially the breakup phenomena in near nozzle are not fully understood yet.

The main breakup of the air assisted atomizer depends on whether the mixing is internal or external. The border between these processes is not clear. In order to study changes in the spray configuration due to the way of mixing, the spray was investigated for the transition from internal to external mixing. Therefore, an air assisted atomizer which allows adjusting the distance between injector to cover was used. The atomizer consists of two components, which are a liquid injector and an injector cover with orifice. The atomization air flows through the gap between the injector and the cover. In this study, the water spray characteristics depending on the air and liquid mixture position were investigated experimentally by two optical measurement techniques.

\section{Material and methods}

In this study, PDA and Direct imaging techniques are used to investigate about the spray breakup. 


\section{Phase Doppler Anemometer}

PDA is an established method to acquire droplet spectra of sprays. Durst et al. [3] showed that the phase shift of the scattered light of laser-Doppler anemometry directly yields the droplet diameter. The phase shift is measured by at least two detectors, which are placed at an off-axis position according to the experimental setup. The receiver angle was set as the recommended value of around $40^{\circ}$ to be higher the signal to noise ratio. The measurement positions are set at $20 \mathrm{~mm}$ and $40 \mathrm{~mm}$ downstream from liquid nozzle exit.

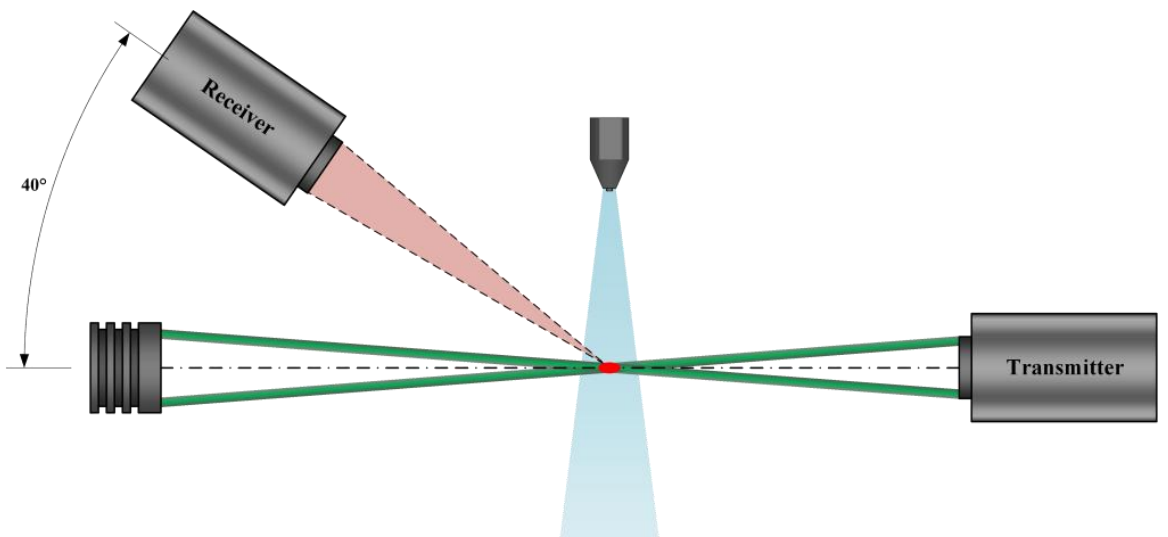

Figure 1. Setup of Phase Doppler Anemometer

\section{Direct droplet imaging}

The direct droplet imaging is based on a shadowgraphy setup and an evaluation algorithm programmed in MATLAB. Kapulla et al. [4] showed by a similar principle in 2007 that droplet imaging is possible for droplets with a diameter down to at least $5 \mu \mathrm{m}$. In order to resolve droplets down to $10 \mu \mathrm{m}$ or even less, magnifications up to 20 and hence strong illumination is needed.

The measurement positions are set at $40 \mathrm{~mm}$ downstream from liquid nozzle exit. Also, same setup was used for investigating the spray structure near nozzle exit with low magnification.

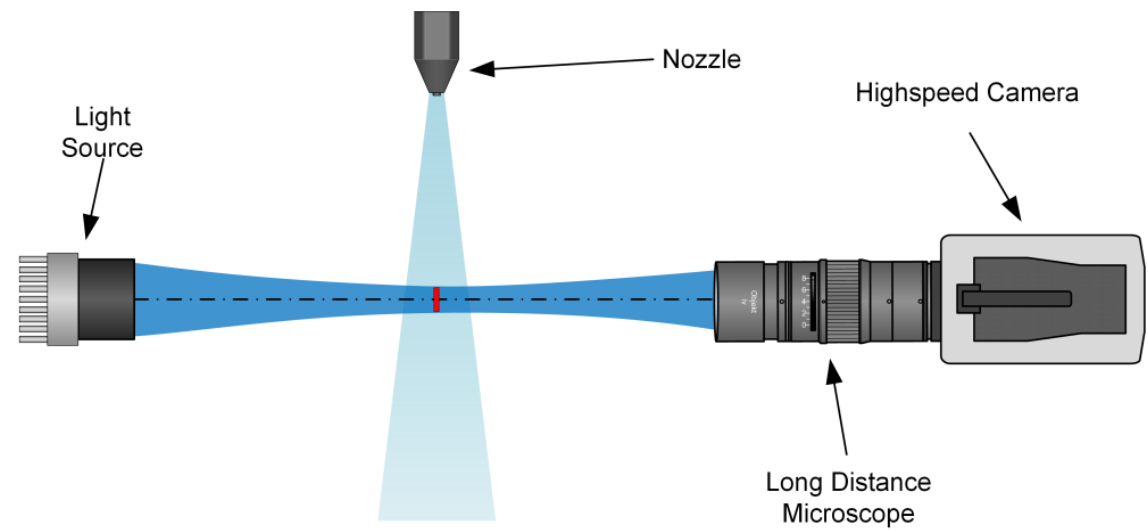

Figure 2. Setup of direct droplet imaging by means of LED light source, long distance microscope and high speed camera

In the setup (Figure 2), light source, measurement volume and camera are located on one axis. A 2.1 W LED is used to illuminate the measurement volume, the shadows of the droplet are captured by a high speed camera (Phantom v711, Vision Research) and focused and enlarged by a long distance microscope (K2-DistaMax, Infinity). The short exposure times of the high speed camera allow grabbing the droplets without unacceptable motion blur. The position and depth of the plane of focus determine which of the droplets are mapped on the sensor of the camera. Therefore the focus adjustment defines the space resolved observation of the spray. Droplets outside the plane of focus either cause a blurred image or only noise if the defocusing is big enough. This relation yields the criterion for recognition of droplets and calculating the diameter. In order to evaluate only the droplets which are within the plane of focus, the local intensity gradient of droplet images is used as criterion for droplet recognition (see Figure 3). 


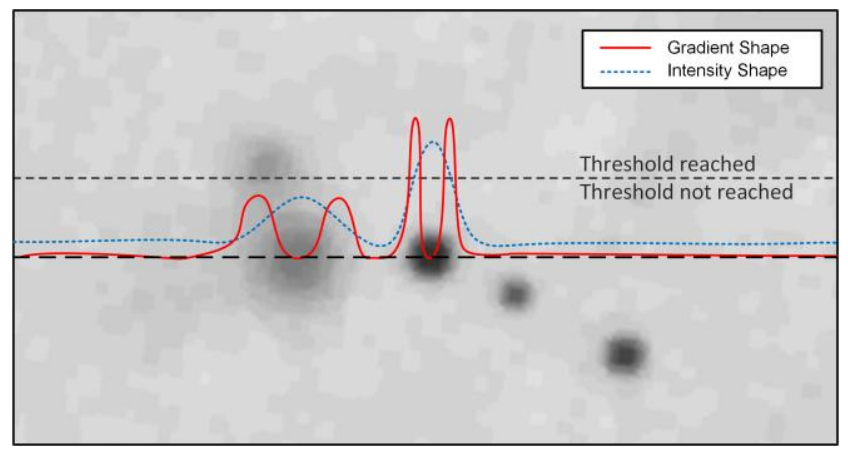

Figure 3. Principle of droplet recognition by threshold values for intensity and intensity gradient

The images are scanned vertically and horizontally for pixels, which fulfil the threshold requirement. Several morphological operations lead to a binary image with potential droplets, which are validated according to circularity and filling factors. Figure 4 refers to a trial measurement of a water spray from same atomizer at $40 \mathrm{~mm}$ downstream from nozzle. Such measurements were used to develop the method. Figure 4 shows the original image (a), the binarized and validated image (b) and the histogram for the results of 1000 images (c).
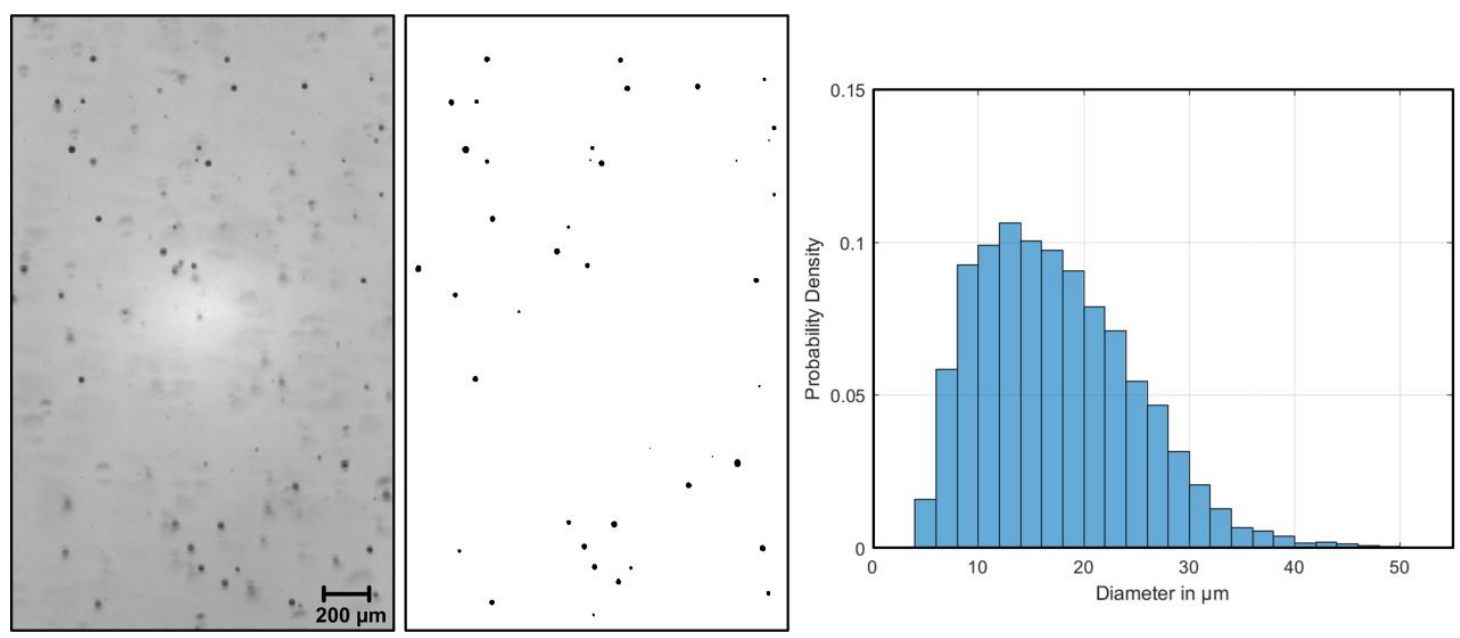

Figure 4. Droplet imaging: raw image (a), binary image (b) and histogram of droplet diameters derived from 1000 images (c)

\section{Air assisted atomizer}

In this study, 3 types of nozzle were used for experiments. Schematic diagram of nozzles are shown in Figure 5. The nozzle has threads for adjusting the distance between injector to cover was used. The atomizer consists of two components, which are a liquid injector and an injector cover with orifice. The atomization air flows through the gap between the injector and the cover. The air channel has swirl generator indicated by a cross in Figure 5 . The position of cover orifice is able to be traversed from the position fully attached to the injector to several millimetre distances from the injector nozzle.

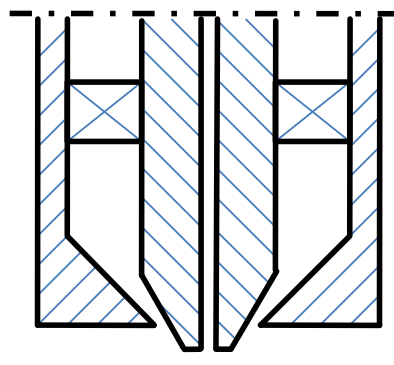

(a) Position 1

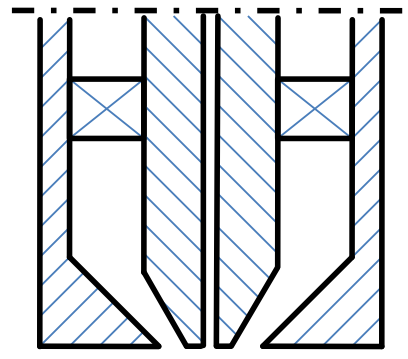

(b) Position 2

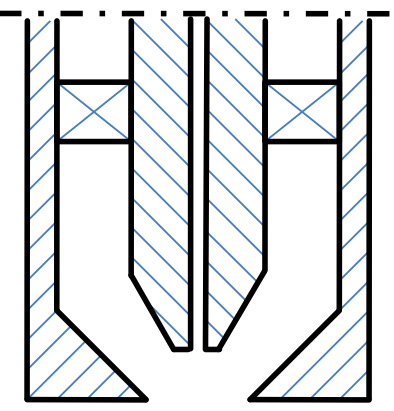

(c) Position 3

Figure 5. Schematic diagram of nozzle cover positions 
The liquid nozzle was fixed and outer cover was adjusted on each measurements. Figure 5(a) shows nozzle cover position 1. Air and liquid are mixed at outside of nozzle (external mixing). Figure 5(b) shows nozzle cover position 2. Exit of air and liquid are in same plane. Figure 5(c) shows nozzle cover position 3. Air and liquid are mixed at inside of outer cover (internal mixing).

\section{Measurement conditions}

Measurements were conducted in room temperature and atmospheric pressure conditions. Two fluids are air and water. 4 constant mass flows of air and water conditions were chosen for operating condition as shown in Table 1. Approximate ratios of air volume flow rate $\left(Q_{a}\right)$ to water volume flow rate $\left(Q_{w}\right)$ are shown in third row.

Table 1. Operating condition of air and water flow rate.

\begin{tabular}{c|ccc}
\hline \hline & Air & Water & $\mathrm{Q}_{\mathrm{a}} / \mathrm{Q}_{\mathrm{w}}$ \\
\hline \hline Condition 1 & $8 \mathrm{~kg} / \mathrm{h}$ & $3 \mathrm{~kg} / \mathrm{h}$ & 2200 \\
Condition 2 & $8 \mathrm{~kg} / \mathrm{h}$ & $6 \mathrm{~kg} / \mathrm{h}$ & 1100 \\
Condition 3 & $14 \mathrm{~kg} / \mathrm{h}$ & $3 \mathrm{~kg} / \mathrm{h}$ & 3800 \\
Condition 4 & $14 \mathrm{~kg} / \mathrm{h}$ & $6 \mathrm{~kg} / \mathrm{h}$ & 1900
\end{tabular}

\section{Results and discussion}

\section{Liquid core and spray structure in near nozzle field}

The liquid core and spray structure in near nozzle field is important information to investigate about breakup phenomena. The high-speed imaging technique was conducted to investigate about the structure. Figure 6 shows the structure in each condition. The field of view was from nozzle exit to around $15 \mathrm{~mm}$ downstream from water nozzle exit. It should be noted that liquid core goes not completely vertical in some conditions are caused by small trajectory error of nozzle when it was fixed.

The liquid core and spray structures depending on the operating conditions on nozzle cover position 1 show relatively small difference compared to that on nozzle cover position 2 . The nozzle cover position 1 has minimum air flow area at nozzle exit. And the velocity at nozzle exit was already reached to subsonic on low mass flow rate of air conditions. Nozzle cover position 2 has larger air flow area at nozzle exit compared to the position 1 and the velocity at nozzle exit did not reached to subsonic at low mass flow rate of air condition. However, the velocity reached to subsonic at high mass flow rate of air conditions. It is understood that the small difference on the liquid core and spray structure of nozzle position 1 was caused by the velocity difference between low mass flow rate of air and high mass flow of that was relatively small compared to the other case.

All pictures on nozzle position 1 and 2 have a liquid core. Despite of condition 2 and 4 , the spray structure on the nozzle cover position 3 have no liquid core on each condition. In the condition 2 and 4, it seems that the liquid core was not completely atomized by swirl air, and the liquid core length on condition 4 was shorter than that of condition 2. The nozzle cover position 3 has largest air flow area at nozzle exit and the predicted velocity at nozzle exits were around $50 \mathrm{~m} / \mathrm{s}$ in condition 2 and around $100 \mathrm{~m} / \mathrm{s}$ in condition 4 . It is suggested that the difference between the liquid core and spray structures on two conditions were caused by the deference between breakups by relative velocity. It seems that the nozzle position 3 had highest sensitivity for $Q_{a} / Q_{w}$ and nozzle position 1 had lowest sensitivity for that.

\section{Spray width against ratio of volume flow rate of air to water}

The spray widths on each condition were compared on measurement plane at 20 and $40 \mathrm{~mm}$. The spray width was obtained from averaged image from 1000 pictures on each condition and position. Figure 7 shows the spray width against the conditions. The solid line shows the results of $20 \mathrm{~mm}$ downstream from water nozzle exit, and the dotted line shows the results of $40 \mathrm{~mm}$ downstream from water nozzle exit. Horizontal axis means ratios of air volume flow rate $\left(Q_{a}\right)$ to water volume flow rate $\left(Q_{w}\right)$. The results of position 1 at $20 \mathrm{~mm}$ and $40 \mathrm{~mm}$ show almost same values on the combinations of $Q_{a} / Q_{w}=1100$ and 2200, and $Q_{a} / Q_{w}=1900$ and 3800. Difference between these combinations is air volume flow rate. It seems that the spray width of nozzle cover position 1 was not affected by $Q_{a} / Q_{w}$ but affected by air flow rate. On the results of position 3 at $20 \mathrm{~mm}$ and $40 \mathrm{~mm}$, there were almost positive correlation between $Q_{a} / Q_{w}$ and spray width. Despite of $Q_{a} / Q_{w}=1100$, the spray widths on nozzle position 3 were wider than that on nozzle position 1. Also, spray widths on nozzle position 2 were between them and a little close to that on position 3. On the condition of $Q_{a} / Q_{w}=1100$, the spray width at $20 \mathrm{~mm}$ of each nozzle cover position were almost same value. This similarity was also shown in Fig.6(b). 


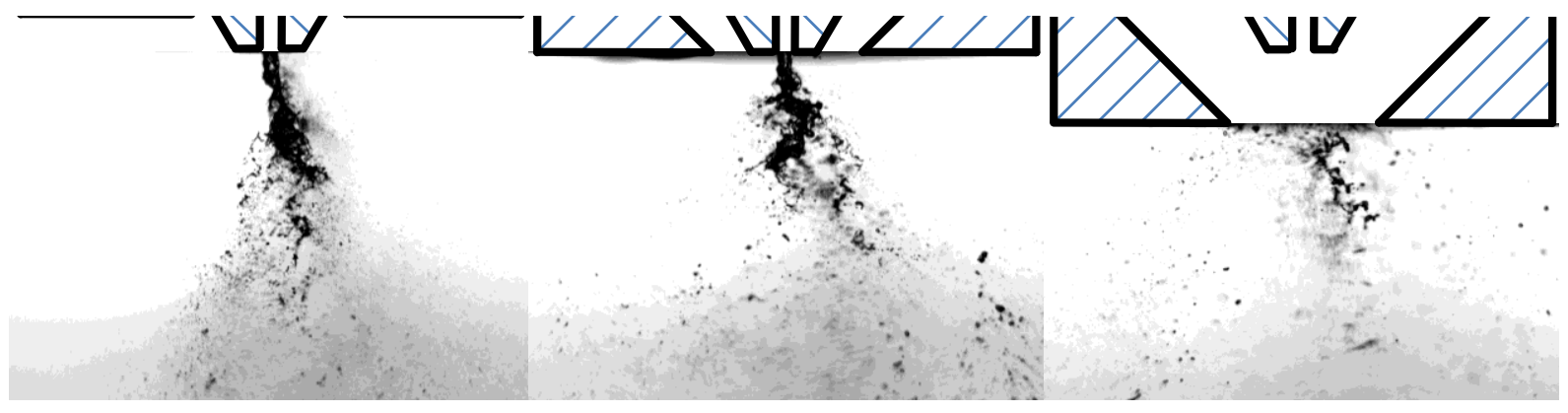

(a) Condition 1: $Q_{a} / Q_{w}=2200$ (Nozzle cover position 1 to 3 from left to right)

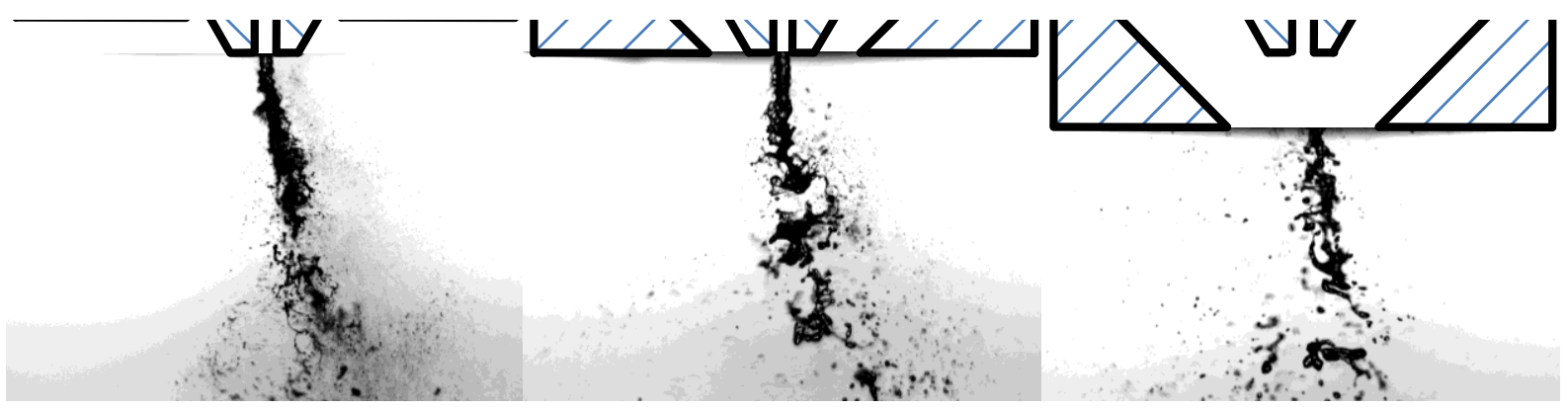

(b) Condition 2: $Q_{a} / Q_{w}=1100$ (Nozzle cover position 1 to 3 from left to right)

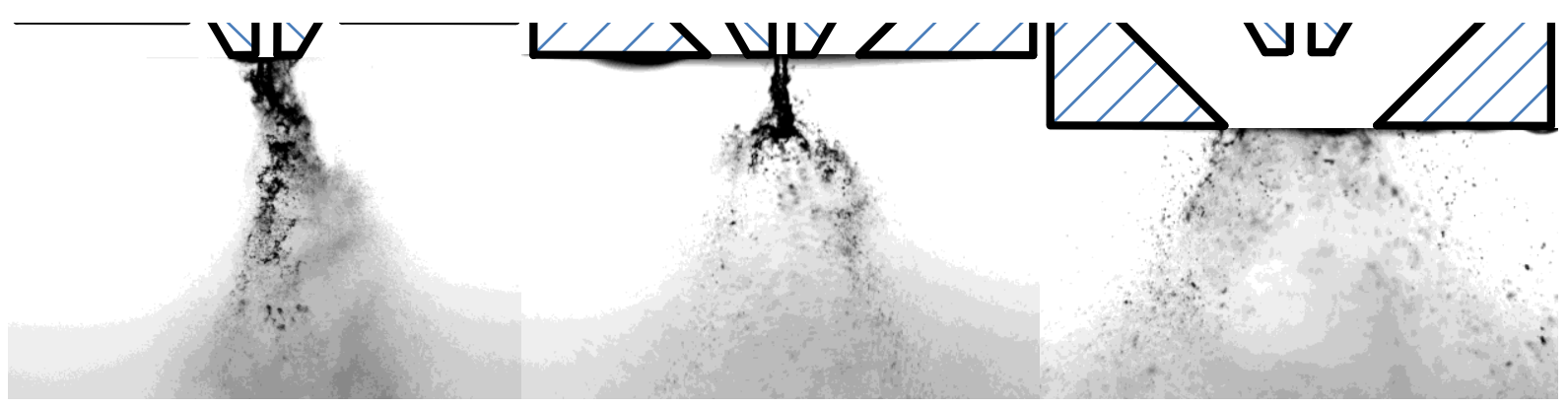

(c) Condition 3: $Q_{a} / Q_{w}=3800$ (Nozzle cover position 1 to 3 from left to right)

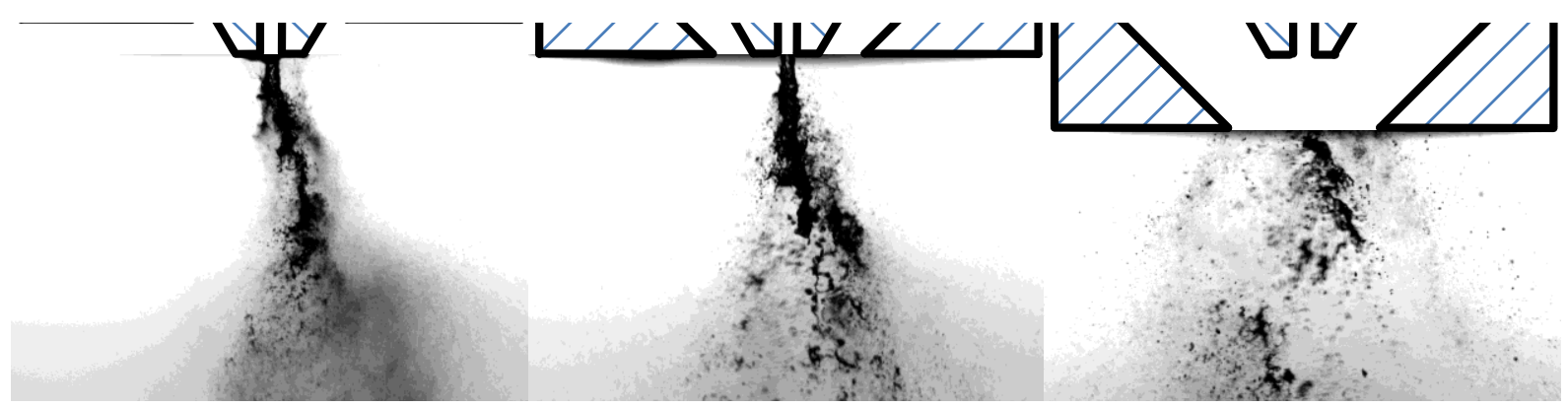

(d) Condition 4: $Q_{a} / Q_{w}=1900$ (Nozzle cover position 1 to 3 from left to right)

Figure 6. Spray structure in near nozzle field. 


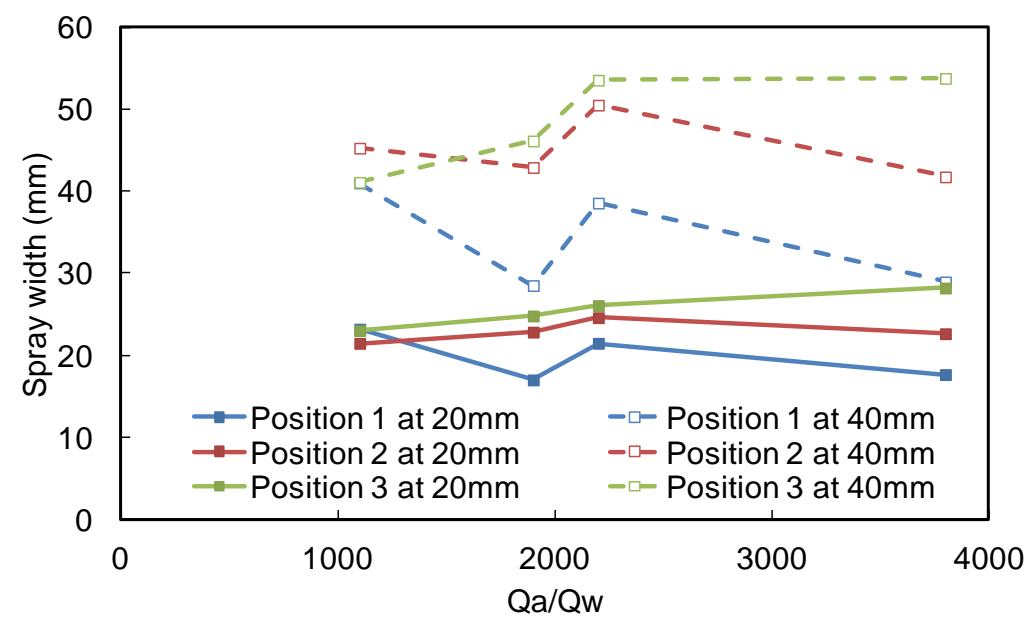

Figure 7. Spray width on each measurement plane

\section{Velocity and diameter of droplets as a function of radial distance}

The arithmetic mean velocity and diameter as a function of radial distance were compared on measurement plane at 20 and $40 \mathrm{~mm}$ on condition 1. These measurements were conducted by PDA around spray centre to middle of centre and periphery to investigate about the breakup phenomena near spray centre. Figure 8(a) shows that the velocities as a function of radial distance on every nozzle cover position. The solid line shows the results of $20 \mathrm{~mm}$ downstream from water nozzle exit, and the dotted line shows the results of $40 \mathrm{~mm}$ downstream from water nozzle exit. Horizontal axis means radial distance from spray centre. The velocities on the nozzle position 1 at $20 \mathrm{~mm}$ downstream shows highest velocity difference between spray centre and $10 \mathrm{~mm}$ radial position. This large velocity difference is also shown on the nozzle position 1 at $40 \mathrm{~mm}$ downstream. As shown in the figure, the velocity distribution on the nozzle position 1 had not so much difference between two measurement planes. The velocities of the nozzle position 2 show the nearly constant value entire the spray at $20 \mathrm{~mm}$ downstream. In case of nozzle position 3 , the velocity decreased around $10 \mathrm{~m} / \mathrm{s}$ near the spray centre with increasing the distance from nozzle exit.

Figure 8 (b) shows that the diameters as a function of radial distance on every nozzle cover position. The droplet diameters were minimum value on spray centre on each position, and the droplet diameters were increased with increasing the distance from spray centre. The droplet diameters decreased on every nozzle cover position with increasing the distance from nozzle, despite of spray centre on the nozzle cover position 1 and 3 . The largest difference between measurement planes on the droplet diameter was occurred on nozzle cover position 2 .

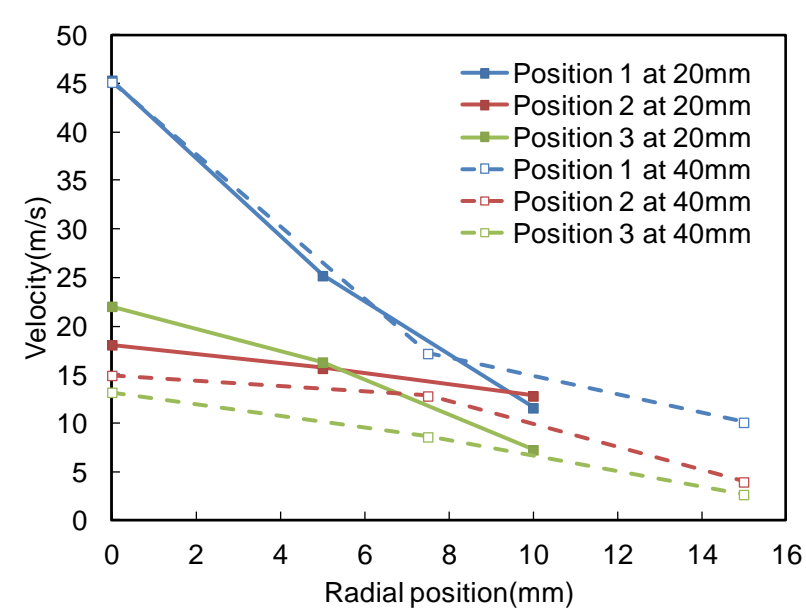

(a) Velocity

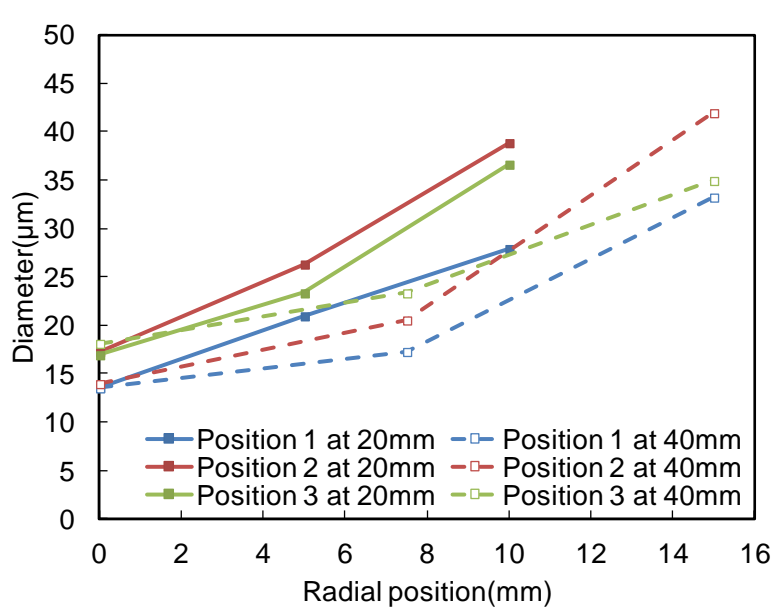

(b) Diameter

Figure 8. Droplet velocity and diameter as a function of radial distance on condition 1 
Table 2. PDA valid rate and RMSD on each measurement position

\begin{tabular}{|c|c|c|c|c|c|c|c|c|c|c|}
\hline \multicolumn{2}{|c|}{ Distance $(\mathrm{mm})$} & \multicolumn{3}{c|}{ PDA valid rate(\%) } & \multicolumn{2}{c|}{ RMSD on velocity $(\mathrm{m} / \mathrm{s})$} & \multicolumn{3}{c|}{ RMSD on diameter $(\mu \mathrm{m})$} \\
\cline { 2 - 11 } & \multicolumn{2}{|c|}{ Nozzle cover position } & \multicolumn{2}{c|}{ Nozzle cover position } & \multicolumn{3}{c|}{ Nozzle cover position } \\
\hline from nozzle & from spray centre & 1 & 2 & 3 & 1 & 2 & 3 & 1 & 2 & 3 \\
\hline \multirow{3}{*}{20} & 0 & 87.81 & 96.70 & 95.31 & 12.04 & 6.63 & 8.48 & 14.36 & 18.46 & 20.97 \\
\cline { 2 - 11 } & 5 & 91.61 & 95.38 & 93.68 & 15.70 & 7.80 & 7.79 & 16.78 & 31.89 & 25.80 \\
\cline { 2 - 11 } & 10 & 96.43 & 92.52 & 88.59 & 10.01 & 7.87 & 5.58 & 16.82 & 39.21 & 32.54 \\
\hline \multirow{3}{*}{40} & 0 & 83.95 & 97.32 & 96.99 & 10.00 & 4.09 & 4.24 & 7.41 & 8.69 & 13.02 \\
\cline { 2 - 11 } & 7.5 & 85.02 & 97.67 & 96.10 & 7.84 & 5.21 & 4.08 & 9.02 & 14.47 & 14.38 \\
\cline { 2 - 11 } & 15 & 87.42 & 85.63 & 89.05 & 8.93 & 3.08 & 2.24 & 16.84 & 26.28 & 24.17 \\
\hline
\end{tabular}

Table 2 shows PDA valid rate and root mean square deviation (RMSD) on each measurement position. In case of nozzle cover position 1, minimum PDA valid rate in same plane were obtained on measurement at spray centre. On the other nozzle cover positions, those were obtained on most outside measurement position. RMSD shows the magnitude of dispersion of the measured data. In this study, RMSD was calculated by following equation.

$$
R M S D=\sqrt{\frac{1}{n} \sum_{i=1}^{n}\left(x_{i}-\bar{x}\right)^{2}}
$$

Here, $n$ means number of measurement data, $x_{i}$ means one of the measurement data, and $\bar{x}$ means averaged value of the data. Relatively large RMSD on velocity were obtained on the measurement of nozzle cover position 1 at $20 \mathrm{~mm}$ from nozzle plane, because of the high arithmetic mean value. Relatively large RMSD on diameter were obtained on the measurement of nozzle cover position 2 at $20 \mathrm{~mm}$ from nozzle plane. The largest RMSD was obtained at the most outside measurement position, and it was almost same as the arithmetic mean diameter of the measurement position. It might be caused by large diameter droplets which were small number but existing as shown in the centre picture of Figure $6(a)$.

\section{Probability density of droplet diameter}

The probability densities of droplet diameter were investigated around spray centre at $40 \mathrm{~mm}$ downstream from nozzle exit by using PDA and direct imaging techniques. These diagnostics performed on difference spray sampling methods, PDA is temporal sampling and direct imaging techniques is spatial sampling. Tropea et al reviewed and explained the several optical measurement techniques[5]. According to the literature, it seems that the differences between two diagnostics are obtained when there are positive or negative relation between droplet velocity and diameter. In this study, the chosen conditions are 2, 3, and 4 of nozzle position 3 , because the structure of spray on these conditions has clear difference between conditions as shown in Figure 6 . In these conditions, there are almost no relation between velocity and diameter of droplets.

Figure 9 shows the probability density distribution of droplet diameter around spray centre. The solid line shows the results from PDA measurement, and the dotted line shows the results of direct imaging techniques. Horizontal axis means diameter. The PDA and direct imaging results on condition 3 have a peak at the same diameter and almost same value. Also, the distribution curve was nearly fit to the others. The PDA results on condition 4 shows slightly different to direct imaging results. On the condition 2, there are relatively high difference between PDA results and direct imaging. As shown in Figure 6, there are huge droplets or ligaments on condition 2, and also there are almost no huge droplets in near spray centre on condition 3 . The diameter measurement principle of PDA is depending on the surface curvature. Therefore, it is understood that the difference between the results of PDA and direct imaging were be caused by the incorrect measurement of bouncing unspherical droplets. 


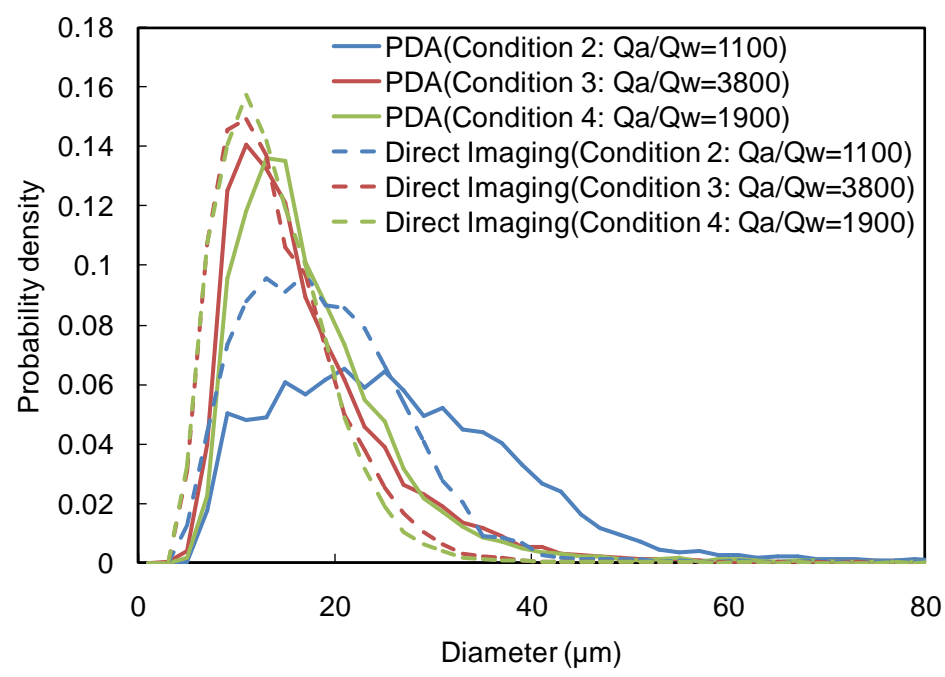

Figure 9. Probability density of droplet diameter around spray centre on nozzle cover position 3

\section{Conclusions}

Imaging techniques were used for taking the spray structure as well as liquid core in near nozzle field. The water mass flow, atomization air mass flow, and the cover position were changed, and their effects on the spray core structure and spray width were investigated. From these investigations, it seems that the nozzle position 3 had highest sensitivity for $Q_{a} / Q_{w}$ and nozzle position 1 had lowest sensitivity for that on spray core structure. And the spray width of nozzle cover position 1 was not affected by $Q_{a} / Q_{w}$ but affected by air flow rate.

Phase Doppler Anemometer (PDA) was applied for measurement of velocity and size of droplets in water spray which injected into the atmosphere. The measurement positions were set at planes which located on 20 and $40 \mathrm{~mm}$ downstream from the nozzle orifice. For each downstream position, radial profiles of the spray pattern were measured on one air to water flow rate condition. The cover positions were changed and the differences on the droplet velocity and diameter as a function of radial distance on each nozzle cover position were discussed. The velocity distribution on the nozzle position 1 had not so much difference between two measurement planes. The droplet diameters were minimum value on spray centre on each nozzle cover position, and the droplet diameters were increased with increasing the distance from spray centre.

Also, high resolution direct imaging technique has been developed and applied for measurement of diameter of droplets. The results from these measurement techniques were compared and they correspond well at the high air to water flow rate conditions. At the low air to water flow rate condition, the agreement of the results of these measurements became lower. From spray picture, there were huge droplets or ligaments. The diameter measurement principle of PDA is depending on the surface curvature. Therefore, it might be caused by the incorrect measurement of bouncing unspherical droplets.

\section{References}

[1] Nukiyama, S., Tanasawa, Y., "An Experiment on the Atomization of Liquid by Means of an Air Stream(the $2^{\text {nd }}$ Report)", Transaction of JSME Vol.4 (15),1939, pp. 138-145.

[2] Lefebvre, A. H., "Twin-fluid atomization: Factors influencing mean drop size", 1991, Proceedings of ICLASS91, pp. 49-64.

[3] Durst, F., Zare, M., "Laser Doppler Measurements in Two-Phase Flow", Proceedings of the LDA Symposium Copenhagen 1975, pp.403-429.

[4] Kapulla, R., Trautmann, M., Hernandez Sanchez, A., Calvo Zaragoza, S., Hofstetter, S., Häfeli, C., Güntay , S., "Droplet size distribution measurements using phase-Doppler anemometry and shadowgraphy: Quantitative comparison", 15. GALA Fachtagung Lasermethoden in der Strömungsmesstechnik 2007.

[5] Tropea, C., "Optical Particle Characterization in Flows", 2011, Annu. Rev. Fluid Mech., 43, pp. 399-426. 